\title{
Enfermedad Gota: Revisión Bibliográfica
}

\section{Gout Disease: Literature Review}

Jacqueline Araya-Ortega, Gilmar Contreras-Chacón, Michelle Herrera-Obando, Luisa Lopez-Vargas, Yerick-Pereira Zumbado, Vera Serrano- Selva. Estudiantes de la facultad de Microbiología, Universidad de Ciencias Médicas de Costa Rica (UCIMED)

Pág. 2,3

Recibido: 17-10-2019

Aceptado: 04-02-2020

\section{Resumen}

La gota es una enfermedad caracterizada por un aumento del ácido úrico, el cual es un producto de desecho del catabolismo de las purinas en humanos y es excretado por la orina. Clínicamente, se presenta como una monoartritis inflamatoria aguda y autolimitada que afecta las articulaciones de la extremidad inferior. En cuanto a su clasificación, se divide en gota primaria y secundaria. Para el diagnóstico, el papel del microbiólogo es fundamental, ya que es el que reporta los cristales de ácido úrico, los cuales, correlacionando la clínica y diversos exámenes, podría llevar a la conclusión de la enfermedad de la gota.

\section{Abstract}

Gout is a disease characterized by an increase in uric acid, which is a waste product of purine catabolism in humans and is excreted in the urine. Clinically it presents as an acute and self-limited inflammatory monoarthritis that affects the joints of the lower limb. As for its classification, it is divided into primary and secondary gout. In the diagnosis, the role of the microbiologist is essential, since it is the one that reports uric acid crystals, which correlating the clinic and various tests could lead to the conclusion of gout disease.

Palabras clave: gota, hiperuricemia, tofos, artritis, monoarticular, eritema, ácido úrico.

Keywords: gout, hyperuricemia, tofos, arthritis, monoartiarticular, erythema, uric acid.

\section{Introducción}

La gota es una enfermedad metabólica autoinflamatoria común, que se caracteriza por una elevación de ácido úrico, llevando a la formación y acumulación de cristales de urato monosódico (MSU) en el líquido sinovial. (Sicras et al., 2012). Generalmente, se presenta como una monoartritis inflamatoria aguda, que afecta las articulaciones de la extremidad inferior. El nivel elevado de urato sérico es el principal factor de riesgo para la deposición de cristales de MSU y el desarrollo de gota. (Dalbeth et al, 2019)

\section{Marco teórico}

La gota es una enfermedad metabólica que se caracteriza por un incremento en las concentraciones corporales de uratos con hiperuricemia sostenida. Afecta predominantemente a hombres en edad adulta, mujeres posmenopáusicas y adultos mayores. (Academia Nacional de Medicina de México, 2014)

\section{Manifestaciones clínicas}

Suele presentarse de forma brusca y los síntomas y signos inflamatorios aparecen entre las 12 o 24 horas. El dolor es intenso, se acompaña de calor, tumefacción e impotencia funcional. Es característico la presencia de eritema, que cuando es extensa puede simular una flebitis o una celulitis. (Dalbeth et al, 2019). La forma más característica es la artritis monoarticular en el metatarso del pie, aunque a veces también se presenta en el talón, en el tobillo o en el empeine. (Lozano, 2004) 


\section{Clasificación etiológica}

Gota primaria: Incluye los trastornos del metabolismo de las purinas idiopáticas o hereditarias que cursan con hiperproducción de ácido úrico y clásicamente es llamada gota metabólica primaria. Además, el componente hereditario se debe al efecto selectivo de la secreción tubular de ácido úrico. (Lozano, 2004)

Gota secundaria: Comprende los casos de gota en los que el origen de la hiperuricemia es adquirido. Para la hiperproducción de ácido úrico, puede darse de origen exógeno. Sin embargo, las causas más frecuentes de gota secundaria son de origen renal. (Lozano, 2004)

\section{Diagnóstico}

El diagnóstico debe asegurar la presencia de los cristales de ácido úrico en el líquido sinovial o en los tofos (Arredondo, 2011).

- Análisis de suero: medir los niveles de ácido úrico y creatinina. Sin embargo, existen casos donde los niveles de ácido úrico se encuentran elevados, pero no presentan enfermedad.

- Prueba de líquido sinovial: extraer líquido de la articulación afectada y se observa si hay presencia de cristales de urato.

- Radiografías de las articulaciones: descartar otras causas de inflamación articular.

- Ecografía músculo-esquelética: detectar cristales de urato en las articulaciones.

- Tomografía computarizada de doble energía: no se realiza de rutina debido al costo. (Mayo Clínica, 2019).

\section{Tratamiento}

Para los recrudecimientos se recomienda el uso de la colchicina. Este medicamento es eficaz al comienzo del ataque. Los antiinflamatorios no esteroides (AINE) son el tratamiento de elección. Además, en los pacientes crónicos se recomienda el uso de corticosteroides. (Bolster, 2019).

\section{Conclusiones}

El ácido úrico es un producto del catabolismo de las purinas, el cual puede jugar un papel patológico en múltiples enfermedades, tanto sistémicas como localizadas. El diagnóstico de la enfermedad es sencillo y se torna vital para la detección a tiempo.

\section{Referencias}

Arredondo, A. (2011). La gota, una antigua enfermedad con un nuevo enfoque. Recuperado de: https://www. portalesmedicos.com/publicaciones/authors/1158/Dr.Alfredo--Arredondo-Bruce?Page=1

Academia Nacional Medicina de México. (2014). Gota. Recuperado de: http:// www.scielo.org. $\mathrm{mx} / \mathrm{scielo} . \mathrm{php}$ ? script $=\mathrm{sci}$ arttext\&pid=S0026-17422014000400058

Bolster, M. (2019). Gota. recuperado de: https:// www.rheumatology.org/l-Am-A/Patient-Caregiver/ Enfermedades-y-Condiciones/Gota

Dalbeth, N., Choi, K.C., Joosten, L., Khanna, P., Matsuo, H., Pérez, F., \& Stamp, K.L. (2019). Gout. Nature Reviews Disease Primers. Recuperado de: https://www.nature.com/articles/s41572-019-0115-y

Lozano, J.A. (2004). Hiperuricemia y gota clasificación, clínica y tratamiento, Recuperado de: https://www. elsevier.es/es-revista-offarm-4-pdf-13061799

Mayo Clinic. (2019). Gota. Diagnóstico y tratamiento. Recuperado de: https://www.mayoclinic.org/es-es/ diseases-conditions/gout/diagnosis-treatment/drc20372903?

Sicras et al. (2012). Estudio de pacientes con gota: características clínicas en los últimos 2 años de seguimiento. Recuperado de:https://www.anmm.org. mx/GMM/2012/n5/GMM_148_2012_5_448-456.pdf 